\title{
Segurança e vulnerabilidade hídrica: evoluções conceituais à luz da gestão Integrada e Sustentável
}

\author{
Water Security and Vulnerability: \\ conceptual evolution in the Integrated and Sustainable Management \\ Seguridad y vulnerabilidad del Agua: \\ evolución conceptual a la Luz de la Gestión Integrada y Sostenible
}

\author{
Micaella Raíssa Falc ão de Moura ${ }^{1}$ \\ Francine Modesto dos Santos ${ }^{2}$ \\ Carlos de Oliveira Galvão ${ }^{3}$ \\ Suzana Maria Gico Lima Montenegro ${ }^{4}$ \\ Simone Rosa da Silva
}

\section{Resumo}

MOURA, M. R. F. de; SANTOS, F. M. dos; GALVÃO, C. de O.; MONTENEGRO, S. M. G. L.; SILVA, S. R. da. Segurança e vulnerabilidade hídrica: evoluções conceituais à luz da Gestão Integrada e Sustentável. Rev. C\&Trópico, v. 44, n. 1, p. 119-141, 2020. DOI: https://doi.org/10.33148/cetropicov44n1(2020)art6

O gerenciamento integrado da água, com premissas baseadas na Sócio-hidrologia, direciona os sistemas socioecológicos à sustentabilidade. O presente artigo apresentou um Mapa Conceitual para compreensão de evoluções conceituais relativas à segurança e à vulnerabilidade hídrica na perspectiva da Sustentabilidade Global. Para isso, realizou-se uma revisão de literatura para o desenvolvimento de uma estrutura teórica que relaciona conceitos-chave fundamentais para análise e discussão interdisciplinar do gerenciamento sustentável da água. Observa-se que existem evoluções conceituais no campo dos recursos hídricos que passaram a contemplar abordagens interdisciplinares que, por muito tempo, foram negligenciadas. $\mathrm{O}$ Mapa Conceitual mostrou ainda que há múltiplos focos no conceito e no estudo da Segurança Hídrica, de modo que esta pesquisa manteve o foco na Vulnerabilidade

\footnotetext{
Doutora em Recursos Hídricos no Programa de Pós-graduação em Eng. Civil e Ambiental do Centro de Tecnologias e Geociências UFPE- (PPGEC-CTG-UFPE). Docente do Centro Universitário Estácio do Recife. E-mail: micaellaraissa@hotmail.com Orcid: https://orcid.org/0000-0002-8710-3429

2 Mestre e Doutora em Demografia pela Universidade Estadual de Campinas (UNICAMP). Realizou pós-doutorado na Universidade Federal de Campina Grande (UFCG) como bolsista do INCT Mudanças Climáticas - Fase II (2018-2020). E-mail: franmodesto@gmail.com Orcid: https://orcid.org/0000-0002-5249-1230

3 Professor Titular da Universidade Federal de Campina Grande. Doutor em Recursos Hídricos e Saneamento Ambiental pela Universidade Federal do Rio Grande do Sul (1999). E-mail: carlos.o.galvao@gmail.com Orcid: https://orcid.org/0000-0002-0800-7085

4 Professora Titular, membro permanente do Programa de Pós- Graduação em Engenharia Civil da UFPE (Mestrado e Doutorado) e do Programa de Pós- Graduação em Engenharia Agrícola e Ambiental da Universidade Federal Rural de Pernambuco. E-mail: suzanam.ufpe@gmail.com Orcid: https://orcid.org/0000-0002-2520-5761

5 Professora Associada da Escola Politécnica da Universidade de Pernambuco, docente permanente do Mestrado em Engenharia Civil e docente colaboradora do Mestrado em Tecnologia Ambiental do ITEP - Instituto de Tecnologia de Pernambuco (ITEP-OS). E-mail: simonerosa@poli.br Orcid: https://orcid.org/0000-0001-7138-7546
} 
e na Sustentabilidade, entendendo que estes são elementos relevantes para consolidar a prática da Gestão Integrada de Recursos Hídricos em prol da garantia da Segurança Hídrica.

Palavras-chave: Segurança Hídrica. Gestão Integrada de Recursos Hídricos. Vulnerabilidade Hídrica. Sus-tentabilidade.

\section{Abstract}

MOURA, M. R. F. de; SANTOS, F. M. dos; GALVÃO, C. de O.; MONTENEGRO, S. M. G. L.; SILVA, S. R. da. Water Security and Vulnerability: Conceptual Evolution in the Integrated and Sustainable Management. Rev. CઐTrópico, v. 44, n. 1, p. 119-141, 2020. DOI: https://doi.org/10.33148/cetropicov44n1(2020)art6

Integrated water management based on Socio-hydrology premises leads socio-ecological systems to sustainability. This article presented a Conceptual Map for understanding conceptual developments related to water security and vulnerability from the perspective of Global Sustainability. To reach this goal, a literature review was carried out to develop a theoretical framework that lists key concepts that are fundamental for the interdisciplinary analysis and discussion of sustainable water management. It was observed that there are conceptual evolutions in the field of water resources that started to contemplate interdisciplinary approaches that, for a long time, were neglected. The Conceptual Map also showed the multiple focuses on the definition and study of Water Security. This research kept the focus on Vulnerability and Sustainability, understanding that they are relevant elements to consolidate the Integrated Water Resources Management practice in favor of guarantee Water Security.

Keywords: Water Security. Integrated Water Resources Management. Water Vulnerability. Sustainability.

\section{Resumen}

MOURA, M. R. F. de; SANTOS, F. M. dos; GALVÃO, C. de O.; MONTENEGRO, S. M. G. L.; SILVA, S. R. da. Seguridad y vulnerabilidad del Agua: Evolución Conceptual a la Luz de la Gestión Integrada y Sostenible. Rev. CひTrópico, v. 44, n. 1, p. 119-141, 2020. DOI: https://doi.org/10.33148/ cetropicov44n1(2020)art6

La gestión integrada del agua con fundamentos basados en la Sociohidrología dirige los sistemas socioecológicos hacia la sostenibilidad. Este artículo presenta un Mapa Conceptual para comprender los desarrollos conceptuales relacionados con la seguridad y vulnerabilidad del agua en la perspectiva de la sostenibilidad global. Con este fin, se realizó una revisión exhaustiva de la literatura para desarrollar un marco teórico que enumera los conceptos clave que son fundamentales para el análisis interdisciplinario y la discusión de la gestión sostenible del agua. Se observó que hay evoluciones conceptuales en el campo de los recursos hídricos que comenzaron a contemplar enfoques interdisciplinarios que, durante mucho tiempo, fueron descuidados. El Mapa Conceptual también muestra que existen 
múltiples enfoques en la definición y el estudio de la Seguridad del Agua. Esta investigación mantuvo el enfoque en Vulnerabilidad y Sostenibilidad, entendiendo que estos son elementos relevantes para consolidar la práctica de la Gestión Integrada de los Recursos Hídricos a favor La garantía de Seguridad del Agua.

Palabras clave: Seguridad del agua. Gestión integrada de recursos hídricos. Vulnerabilidad del agua. Sostenibilidad.

Data de submissão: $18 / 04 / 2020$

Data de aceite: 08/06/2020

\section{Introdução}

O reconhecimento da água como direito humano fundamental é ainda recente e a proteção deste direito exige que modelos de gestão essencialmente pragmáticos e higienistas deem lugar a abordagens interdisciplinares fundamentadas na ciência Sócio-hidrológica. Um grande diferencial desta ciência, quando comparada às demais ciências interdisciplinares que lidam com a água, é a inclusão direta e bidirecional de feedbacks entre os sistemas hídrico e humano. Nesse sentido, a Sócio-hidrologia dá suporte à prática da chamada Gestão Integrada de Recursos Hídricos (GIRH) (SIVAPALAN et al., 2012; 2014).

Segundo Cook e Bakker (2012), tanto a GIRH quanto a Segurança Hídrica $(\mathrm{SH})$ podem ser consideradas paradigmas complementares, pois implicam a necessidade de integrar quantidade e qualidade da água, considerando aspectos sociais e ecossistêmicos. Beek e Arriens (2014) reforçam que a SH pode ser compreendida como o objetivo principal da Gestão Integrada. Tais conceitos, porém, abrigam intensa complexidade quanto à sua aplicação prática, tendo em vista que os múltiplos usos e demandas por água desafiam a capacidade dos sistemas de governança em oferecer respostas (RIBEIRO; FORMIGA-JOHNSSON, 2018).

Apesar dos esforços para consolidar a prática da GIRH em prol da garantia da $\mathrm{SH}$, observam-se entraves à operacionalização desses conceitos. O mesmo problema pode ser notado com relação ao conceito de Vulnerabilidade Hídrica (VH). Nesse sentido, Grosbois e Plummer (2015) afirmam que a avaliação da VH contribui para uma sinalização mais direta acerca das insuficiências, das ausências ou das fragilidades relacionadas aos recursos hídricos e à sua correlação com outros sistemas em uma determinada região.

No âmbito hidrológico brasileiro, as noções de vulnerabilidade permeiam ordenamentos jurídicos diversos (e.g. Plano Nacional de Segurança Hídrica PNSH - 2019). Contudo, em razão de fatores como a não efetividade de dispositivos expressos na própria Lei das Águas 9433/97 (BRASIL, 1997), o cenário de vulnerabilidade hídrica tem sido atestado em relatórios da Agência Nacional de Águas (ANA) e em pesquisas científicas. Os documentos da ANA caracterizam 
o quadro nacional de Insegurança Hídrica (IH), seja pela falta do recurso hídrico (cenário de secas) ou por seu excesso (cenário de cheias) (DAMACENA, 2015; BOLSON; HAONAT, 2016).

No cenário de secas, o quadro nacional de IH tem sido abordado principalmente na região do semiárido. As previsões de intensos extremos climáticos e a degradação dos solos aumentam as incertezas nos processos administrativos da água, colaborando para uma maior suscetibilidade aos danos causados por secas mais intensas e prolongadas (MARENGO, 2008; MARENGO et al., 2011). Destacam-se as particularidades fisioclimáticas desta região, que, aliadas a políticas públicas equivocadas, culminaram para acentuar esta conjuntura crítica de elevada vulnerabilidade (CIRILO, 2008; CIRILO; MONTENEGRO; CAMPOS, 2017).

Dessa forma, faz-se necessário que as complexidades e os conceitos intrínsecos aos processos de gerenciamento das águas sejam analisados com clareza, pois permitem trazer à tona obstáculos à consolidação de políticas públicas, por exemplo, possibilitando ainda encontrar oportunidades à reestruturação do sistema e ao fortalecimento de medidas adaptativas (CARVALHO; CURI, 2016; LOPEZ PORRAS; STRINGER; QUINN, 2019).

Diante do exposto, este artigo desenvolve uma estrutura teórica (Mapa Conceitual) para compreensão de evoluções conceituais relativas à segurança e à vulnerabilidade hídrica na perspectiva da sustentabilidade, além de realizar uma análise na esfera estadual, sendo identificados marcos político-institucionais responsáveis por introduzir formalmente o conceito de segurança hídrica no estado de Pernambuco, localizado na região Nordeste do Brasil.

O primeiro passo para alcançar o objetivo proposto consistiu em uma revisão abrangente da literatura para a formulação do embasamento teórico em acordo com a temática relacionada a: a) Sustentabilidade Global (e.g. UN, 2015); b) Sócio-hidrologia (e.g. SIVAPALAN et al., 2014; MAO et al., 2017); c) Gestão Integrada de Recursos Hídricos - GIRH (e.g. SAVENIJE; VAN DER ZAAG, 2008; GWP-C, 2015); d) SH (e.g. GWP, 2000; OECD, 2013)); e e) Vulnerabilidade (e.g. ADGER, 2006; SULLIVAN, 2011; PLUMMER; de LOË; ARMITAGE, 2012). Para tanto, foram reunidos e catalogados para análise artigos científicos, instrumentos jurídicos e outros documentos nacionais e internacionais.

Esses conceitos-chave possibilitam um melhor entendimento das discussões interdisciplinares apontadas como fundamentais para analisar a segurança e a vulnerabilidade da água. A partir da revisão da literatura, foi desenvolvida a estrutura teórica (Mapa conceitual da SH e da VH na perspectiva da Sustentabilidade Global).

\section{Segurança Hídrica (SH)}

Devido às perdas progressivas e generalizadas de serviços ecológicos, alcançar a segurança hídrica a partir de uma perspectiva de desenvolvimento sustentável requer um compromisso global significativo para redirecionar a trajetória 
descendente dos ecossistemas e promover níveis coletivos de gerenciamento de recursos naturais. Até o início dos anos 90, o conceito de SH estava significativamente focado em infraestruturas hidráulicas para conciliar demandas e disponibilidade de água, contando com soluções de engenharia centralizadas e sem considerar ou priorizar medidas de prevenção com boa relação custo-benefício (VÖRÖSMARTY et al., 2018; GLEICK, 1993, 2002).

Nesse sentido, Gleick (2002, p.373) traz o conceito de caminhos rígidos ou "hard paths" e caminhos flexíveis da água ou "soft water paths": o primeiro depende quase que exclusivamente da infraestrutura centralizada para captar, tratar e fornecer suprimentos de água, enquanto o segundo complementa o primeiro, investindo em instalações descentralizadas, tecnologias, políticas eficientes e capital humano

Esse conceito de $\mathrm{SH}$, no entanto, evoluiu substancialmente, passando a considerar análises multifatoriais que vão de encontro às visões tradicionalistas, nas quais as relações interdisciplinares e transdisciplinares costumavam ser negligenciadas (COOK; BAKER, 2012; JAMES; SHAFIEE-JOOD, 2017; BOGARDI et al., 2012). As mudanças crescentes no clima e no uso e ocupação do solo tornaram a gestão da água uma tarefa difícil, acrescentando variabilidade hidrológica e incerteza aos processos de tomada de decisão (UNESCO, 2013). Nesse contexto, o conceito de SH também está evoluindo para acompanhar as complexas interações entre seres humanos e o meio ambiente.

Consequentemente, os desafios para alcançar a segurança da água assumiram uma dimensão global entre os governos, considerando fundamentalmente a sustentabilidade das nações. Esse fato contribuiu para o surgimento de novas estruturas conceituais que abordam a natureza inter-relacionada dos recursos globais, e exemplo do nexo água-energia-alimento. Esses três eixos estão sob pressão constante, considerando sua importância em sustentar a existência e a sobrevivência da vida humana, conforme apontado por Pasqual, Bollmann e Scott (2016), Pasqual, Lardizabal e Herrera (2015) e Rasul e Sharma (2014).

Os conceitos de segurança hídrica formulados por organizações internacionais incluem a importância da água para atender às demandas humanas essenciais de subsistência. Além da ONU, organizações como World Water Council, Global Water Partnership e WaterAid exploram abordagens quantitativas e qualitativas da disponibilidade de água para as necessidades humanas básicas, destacando o papel de proteção do ecossistema e sua relevância na melhoria da vida das sociedades (UN WATER, 2013; WWC, 2012; GWP, 2014; WATERAID, 2012). A abordagem baseada no risco da água é contemplada pela Organização para a Cooperação e Desenvolvimento Econômico (OCDE), que também considera os riscos responsáveis pelo enfraquecimento da resiliência dos sistemas de água doce (OECD, 2013).

Enquadramentos de múltiplas perspectivas para a $\mathrm{SH}$, em ampla revisão de literatura, são apresentados por Hoekstra, Buurman e Van Ginkel (2018), sublinhando-se que diferentes contextos contribuem para diferentes interpretações do termo. Destaca-se a atenção especial que tem sido dada às questões de segurança hídrica 
urbana (SHU), com estruturas integradas para abordagens quantitativas aplicáveis às cidades em todo o mundo (KRUEGER; RAO; BORCHARDT, 2019), modelos de avaliação da SHU baseados na teoria de catástrofes (YANG et al., 2012) e métodos de seleção de indicadores para medir a SHU de cidades ao longo do tempo (JENSEN; WU, 2018).

No que se refere a índices de segurança hídrica, muitos têm sido diretamente relacionados ao Objetivo de Desenvolvimento Sustentável da água (ODS-6). Gain, Giupponi e Wada (2016) usam uma estrutura de análise espacial de múltiplos critérios para fornecer uma primeira compreensão do status global de segurança hídrica. Os autores apresentam como meta monitorar o progresso em direção aos ODS em diferentes países (e.g. China, Índia, Austrália e Brasil), levando em consideração três dimensões principais (física, socioeconômica e governança) e agregando os indicadores de quatro critérios principais: 'disponibilidade', 'acessibilidade aos serviços', 'segurança e qualidade' e 'gestão'.

Métricas de SH baseadas em ODS também foram propostas por Van Noordwijk et al., (2016). Por meio de um estudo de caso na Indonésia, os autores afirmam que os ciclos de aprendizado adaptável provavelmente direcionam o progresso em direção aos ODS, supondo que a maneira como as pessoas e os ecossistemas possam adaptar-se às mudanças climáticas seja a chave do conceito de segurança. O Quadro 1 apresenta um resumo dos conceitos de segurança hídrica abordados por órgãos internacionais e pela Agência Nacional de Águas (ANA).

Quadro 1: Principais conceitos de segurança hídrica.

\begin{tabular}{|c|c|}
\hline Fonte & Conceito de Segurança Hídrica \\
\hline $\begin{array}{c}\text { WaterAid } \\
(2012)\end{array}$ & $\begin{array}{l}\text { O acesso confiável à água em quantidade suficiente e de qualidade para as neces- } \\
\text { sidades básicas humanas, em pequena escala, garantia dos meios de subsistência e } \\
\text { dos serviços dos ecossistemas locais, juntamente a uma adequada gestão dos riscos } \\
\text { inerentes aos desastres relacionados com a água. }\end{array}$ \\
\hline ONU (2013) & $\begin{array}{c}\text { A capacidade de uma população de salvaguardar o acesso sustentável a quanti- } \\
\text { dades adequadas de água de qualidade para garantir meios de sobrevivência, o } \\
\text { bem-estar humano, o desenvolvimento socioeconômico; para assegurar proteção } \\
\text { contra poluição e desastres relacionados à água, e para preservação de ecossistemas } \\
\text { em um clima de paz e estabilidade política. }\end{array}$ \\
\hline $\begin{array}{r}\text { OECD } \\
(2013)\end{array}$ & $\begin{array}{c}\text { Gerir riscos associados à água, incluindo riscos de armazenamento } \\
\text { de água, excesso, poluição e riscos de enfraquecer ou debilitar } \\
\text { a resiliência dos sistemas de água doce. }\end{array}$ \\
\hline $\begin{array}{l}\text { World Water } \\
\text { Council } \\
\text { (2013) }\end{array}$ & $\begin{array}{l}\text { Consiste, inicialmente, na garantia de necessidades essenciais do dia a dia, como } \\
\text { saúde e alimento: água para produzir produtos alimentícios e melhorar rendimentos } \\
\text { agrícolas; água limpa e segura para ajudar a reduzir doenças de veiculação hídrica- } \\
\text { que continuam a ser uma das principais causas de morte. Consiste ainda na garantia } \\
\text { de segurança econômica e social para produção de bens e serviços necessários ao } \\
\text { desenvolvimento e aumento da qualidade de vida. Também abrange a segurança } \\
\text { ecológica para retornar à natureza o papel essencial da água para preservação da } \\
\text { biodiversidade e manutenção de ecossistemas. }\end{array}$ \\
\hline
\end{tabular}




\begin{tabular}{|c|c|c|}
\hline $\begin{array}{c}\text { Global Water } \\
\text { Partnership - } \\
\text { GWP (2014) }\end{array}$ & $\begin{array}{c}\text { A essência da segurança hídrica é que o interesse pelo recurso base está acompa- } \\
\text { nhado do interesse ao serviço que explora ou utiliza o recurso base, como o uso } \\
\text { humano, agricultura, atividades econômicas e proteção ambiental. Ambos aspectos } \\
\text { de qualidade e quantidade de água devem ser considerados uma vez que a qualida- } \\
\text { de afeta o valor da água e o impacto ao meio ambiente. Segurança hídrica significa } \\
\text { aproveitar o potencial hídrico e combater os efeitos destrutivos da água; ou seja, os } \\
\text { danos causados por inundações, secas, deslizamentos de terra, erosão, poluição e } \\
\text { transmissão de doenças. }\end{array}$ \\
\hline OECD|GWP & $\begin{array}{c}\text { O objetivo da segurança hídrica é aproveitar as oportunidades e gerenciar os ris- } \\
\text { cos associados à água e, ao fazê-lo, promover o crescimento sustentável e maior } \\
\text { bem-estar. }\end{array}$ \\
\hline ANA (2015) & $\begin{array}{c}\text { Condição que visa garantir quantidade e qualidade aceitável de água para abas- } \\
\text { tecimento, alimentação, preservação de ecossistemas e demais usos, associados a } \\
\text { um nível aceitável de riscos relacionados com a água para as pessoas, economias } \\
\text { e meio ambiente. }\end{array}$ \\
\hline
\end{tabular}

Fonte: Adaptado de Melo e Formiga-Johnsson (2017).

Na escala nacional, observa-se, por meio do exposto no Quadro 1, que a ANA segue os moldes e as tendências das organizações internacionais ao consolidar uma abordagem conceitual relativa à segurança hídrica no quadro da gestão dos recursos hídricos brasileiros. Porém, embora os objetivos de assegurar a quantidade e a melhoria da qualidade de água tenham sido contemplados na Política Nacional de Recursos Hídricos, o conceito de segurança da água é recente e carece ainda de legitimidade. Nesse sentido, coloca-se em evidência o Projeto de Lei no 65, de 2017, do Senado Federal, que propôs incluir a SH como um dos pilares da Lei das Águas e incluir os Planos de Segurança Hídrica entre os instrumentos da Política Nacional de Recursos Hídricos (BRASIL, 2017a).

Ainda na esfera nacional, faz-se importante destacar o papel do Plano Nacional de Segurança Hídrica (PNSH), lançado em 2019, fruto de parceria entre a ANA e o Ministério do Desenvolvimento Regional (MDR). O PNSH se configura como iniciativa inédita no país e se alinha com os conceitos internacionais de $\mathrm{SH}$, visando, entre outros, assegurar "um planejamento integrado e consistente de infraestrutura hídrica com natureza estratégica e relevância regional, até o horizonte de 2035, para redução dos impactos de secas e cheias" (ANA, 2019a, p.17).

O PNSH concebeu o Índice de Segurança Hídrica (ISH), com o intuito de "retratar, com simplicidade e clareza, as diferentes dimensões da segurança hídrica, incorporando o conceito de risco aos usos da água" (ANA, 2019a, p.20). Assim como o ISH, indicadores compostos relacionados à SH têm sido eficazes ao utilizar parâmetros técnicos que permitem traçar panoramas da segurança dos recursos hídricos, especialmente em áreas urbanas e em bacias hidrográficas em geral (e.g. RIBEIRO; PIZZO, 2011; TUCCI, 2017; DUTRA, 2017; WANG; LI; LI, 2018; MACHADO, 2018).

Partindo para uma abordagem da esfera estadual, em Pernambuco, nordeste do Brasil, pode-se verificar, por meio de uma análise contextual histórica, que houve uma consolidação de marcos político-institucionais responsáveis por introduzir 
formalmente o conceito de segurança hídrica. A preocupação em expandir os serviços de abastecimento de água e esgotamento sanitário passou a agregar fatores relativos à melhoria da eficiência desses serviços e da gestão institucional, especialmente a partir do ano de 2010. No que se refere à legislação, o Quadro 2 reúne os principais instrumentos legais de Pernambuco que guardam relação com o tema.

Quadro 2: Instrumentos legais de Pernambuco relacionados à temática de segurança hídrica

\begin{tabular}{|c|c|c|}
\hline Ano & $\begin{array}{l}\text { Instrumento Legal } \\
\text { PE }\end{array}$ & Descrição da matéria \\
\hline 1971 & $\begin{array}{l}\text { Lei Estadual } 6.307 \\
29 \text { de julho de } 1971\end{array}$ & $\begin{array}{l}\text { Autoriza o Poder Executivo a constituir a Companhia Pernam- } \\
\text { bucana de Saneamento (COMPESA) e dá outras providências. }\end{array}$ \\
\hline 1994 & $\begin{array}{l}\text { Decreto } n^{\circ} 18.251 \\
21 \text { de dez de } 1994\end{array}$ & $\begin{array}{c}\text { Aprova o Regulamento Geral do Fornecimento de Água e da } \\
\text { Coleta de Esgotos, realizadas pela Companhia Pernambucana } \\
\text { de Saneamento - COMPESA. }\end{array}$ \\
\hline \multirow{3}{*}{1997} & $\begin{array}{l}\text { Lei no } 11.426 \\
17 \text { janeiro de } 1997\end{array}$ & $\begin{array}{c}\text { Dispõe sobre a Política e o Plano Estadual de Recursos Hídri- } \\
\text { cos, institui o Sistema Integrado de Gerenciamento de Recursos } \\
\text { Hídricos e dá outras providências. }\end{array}$ \\
\hline & $\begin{array}{l}\text { Lei } \mathrm{n}^{\circ} 11.427 \\
17 \text { de janeiro de } 1997\end{array}$ & $\begin{array}{c}\text { Dispõe sobre a conservação e a proteção das águas subterrâneas } \\
\text { no Estado de Pernambuco e dá outras providências. }\end{array}$ \\
\hline & $\begin{array}{l}\text { Decreto } n^{\circ} 20.269 \\
24 \text { de dez de } 1997\end{array}$ & $\begin{array}{c}\text { Regulamenta a Lei 11426/1997 que dispõe sobre a Política } \\
\text { Estadual de Recursos Hídricos e o Plano Estadual de Recursos } \\
\text { Hídricos e institui o SIGRH. }\end{array}$ \\
\hline \multirow[b]{2}{*}{1998} & $\begin{array}{l}\text { Decreto } n^{\circ} 20.423 \\
26 \text { de março de } 1998\end{array}$ & $\begin{array}{l}\text { Regulamenta a Lei n } 11.427 \text { de } 17 \text { de janeiro de } 1997 \text { que dispõe } \\
\text { sobre a conservação e a proteção das águas subterrâneas no Estado. }\end{array}$ \\
\hline & $\begin{array}{l}\text { Decreto } n^{\circ} 20.786 \\
10 \text { de agosto de } 1998 \text {. }\end{array}$ & $\begin{array}{l}\text { Aprova o Regulamento do Código Sanitário do Estado de } \\
\text { Pernambuco. Realiza a execução da política governamental de } \\
\text { abastecimento de água e esgotamento sanitário. }\end{array}$ \\
\hline 1999 & $\begin{array}{c}\text { Lei } \mathrm{n}^{\circ} 11.629 \\
28 \text { de janeiro de } 1999\end{array}$ & $\begin{array}{l}\text { Dispõe sobre a estrutura organizacional do Poder Executivo Es- } \\
\text { tadual, cria e extingue cargos e dá outras providências. Criação } \\
\text { Secretaria de Recursos Hídricos (SRH), extinta pela Lei comple- } \\
\text { mentar nº } 49 \text { de } 31 \text { de janeiro de } 2003 \text { e novamente criada pela } \\
\text { Lei } 13.205 \text {, de } 19 \text { de janeiro de } 2007 \text {. }\end{array}$ \\
\hline 2000 & $\begin{array}{l}\text { Portaria SRH no } 2506 \\
\text { de junho de } 2000\end{array}$ & $\begin{array}{l}\text { Estabelece distância mínima entre poços tubulares nas Bacias } \\
\text { Sedimentares Costeiras de Pernambuco, define critérios e limi- } \\
\text { tes para captação de água subterrânea. }\end{array}$ \\
\hline 2003 & $\begin{array}{l}\text { Lei } n^{\circ} 12.505 \\
16 \text { de dez de } 2003\end{array}$ & $\begin{array}{c}\text { Cria o Quadro de Servidores e Empregados da Agência Estadu- } \\
\text { al de Meio Ambiente e Recursos Hídricos - CPRH, e dá outras } \\
\text { providências. }\end{array}$ \\
\hline 2005 & $\begin{array}{l}\text { Lei } n^{\circ} 12.984 \\
30 \text { de dez de } 2005\end{array}$ & $\begin{array}{c}\text { Dispõe sobre a Política Estadual de Recursos Hídricos e o Sis- } \\
\text { tema Integrado de Gerenciamento de Recursos Hídricos, e dá } \\
\text { outras providências. }\end{array}$ \\
\hline 2009 & $\begin{array}{c}\text { Resolução CRH no } \\
005 / 2009-\end{array}$ & $\begin{array}{l}\text { Dispõe sobre aprovação de Projetos de conservação, proteção e } \\
\text { recuperação dos Recursos Hídricos pelos Comitês de Bacias Hi- } \\
\text { drográficas/COBHS e Conselhos Gestores de Açudes/CONSUS } \\
\text { no Estado de Pernambuco e dá outras providências. }\end{array}$ \\
\hline
\end{tabular}




\begin{tabular}{|c|c|c|}
\hline \multirow{4}{*}{2010} & $\begin{array}{l}\text { Lei } n^{\circ} 14.028 \\
26 \text { de março de } 2010\end{array}$ & $\begin{array}{l}\text { Cria a Agência Pernambucana de Águas e Clima - APAC, para } \\
\text { complementar o SIGRH e fortalecer o planejamento e regulação } \\
\text { dos usos múltiplos dos recursos hídricos e dá outras providências. }\end{array}$ \\
\hline & $\begin{array}{l}\text { Lei } n^{\circ} 14.090 \\
17 \text { de junho de } 2010\end{array}$ & $\begin{array}{l}\text { Institui a Política Estadual de Enfrentamento às Mudanças } \\
\text { Climáticas de Pernambuco, e dá outras providências. * Plano } \\
\text { Estadual de Mudanças Climáticas (2011). }\end{array}$ \\
\hline & $\begin{array}{l}\text { Lei } n^{\circ} 14.091 \\
17 \text { de junho de } 2010\end{array}$ & $\begin{array}{l}\text { Institui a Política Estadual de Combate à Desertificação e Miti- } \\
\text { gação dos Efeitos da Seca, e das outras providências. }{ }^{*} \text { Progra- } \\
\text { ma Estadual de Combate à Desertificação e Mitigação }\end{array}$ \\
\hline & $\begin{array}{l}\text { Lei } n^{\circ} 14.258 \\
23 \text { de dez de } 2010\end{array}$ & $\begin{array}{c}\text { Institui a Política Estadual de Gerenciamento Costeiro, e dá ou- } \\
\text { tras providências. Tem por objetivo geral disciplinar e orientar a } \\
\text { utilização dos recursos naturais da Zona Costeira do Estado de } \\
\text { Pernambuco. }\end{array}$ \\
\hline \multirow{3}{*}{2012} & $\begin{array}{l}\text { Decreto } \mathrm{n}^{\circ} 38.147 \text { de } 4 \\
\text { de maio de } 2012\end{array}$ & $\begin{array}{l}\text { Estabelece os procedimentos para realização de aquisições e } \\
\text { contratação de obras e serviços necessários à execução das } \\
\text { ações emergenciais de combate aos efeitos da estiagem no âmbi- } \\
\text { to do Estado de Pernambuco nos anos de } 2011 \text { e } 2012 \text {. }\end{array}$ \\
\hline & $\begin{array}{l}\text { Resolução CRH no } \\
0517 \text { de outubro de } \\
2012\end{array}$ & $\begin{array}{l}\text { Cria e nomeia Grupo de Trabalho para normatização de explora- } \\
\text { ção de areia em leito seco de rios intermitentes em Pernambuco. }\end{array}$ \\
\hline & $\begin{array}{l}\text { Decreto } \mathrm{n}^{\circ} 38.752 \\
22 \text { de Out de } 2012\end{array}$ & $\begin{array}{l}\text { Estabelece procedimentos administrativos de fiscalização do } \\
\text { uso de recursos hídricos no Estado de Pernambuco, e dá outras } \\
\text { providências. }\end{array}$ \\
\hline 2013 & $\begin{array}{l}\text { Lei } \mathrm{n}^{\circ} 14.922 \\
18 \text { de março de } 2013\end{array}$ & $\begin{array}{l}\text { Institui a Política Estadual de Convivência com o Semiárido. } \\
{ }^{*} \text { Plano Estadual de Convivência com o Semiárido* }\end{array}$ \\
\hline \multirow[t]{2}{*}{2017} & $\begin{array}{l}\text { Resolução CRH n02 } \\
03 \text { de maio de } 2017\end{array}$ & $\begin{array}{l}\text { Estabelece normas e procedimentos para obtenção de outorgas } \\
\text { do direito de uso dos recursos hídricos em aluviões localizadas } \\
\text { em rios intermitentes no Estado de Pernambuco, tendo em vista } \\
\text { a exploração de areias. }\end{array}$ \\
\hline & $\begin{array}{l}\text { Portaria Conjunta } \\
\text { APAC/CPRH n } \\
\quad 001 / 2017\end{array}$ & $\begin{array}{l}\text { Estabelecem condições e procedimentos para obtenção da } \\
\text { Licença Ambiental e da Outorga do Uso dos Recursos Hídricos } \\
\text { nos mananciais de domínio do Estado de Pernambuco. }\end{array}$ \\
\hline
\end{tabular}

Fonte: Elaboração dos autores.

\section{Vulnerabilidade Hídrica (VH)}

O conceito de vulnerabilidade pode ser considerado como uma ferramenta analítica eficiente para descrever estado de vulnerabilidade ao dano, prejuízo e impotência de sistemas socioecológicos (ADGER, 2006).

De acordo com Rotava (2014), muitos são os obstáculos e as implicações no que diz respeito à obtenção de um conceito geral para a vulnerabilidade. Embora alguns autores (CUTTER, 1996; HOGAN e MARANDOLA JR., 2006; ROTAVA, 2014) se esforcem para mostrar a multidimensionalidade da vulnerabilidade e a evolução do conceito ao longo do tempo, ainda há um grande desafio, primeiro a) conceitual 
em apreender a multidimensionalidade deste conceito polissêmico e multivariado e, segundo, b) consensual para conceituar vulnerabilidade e, consequentemente, a sua operacionalização (ALMEIDA, 2012).

Marandola Jr. e D'antona (2014) problematizam o conceito de vulnerabilidade apontando os principais entraves para a sua operacionalização e potenciais aplicações, especialmente em políticas públicas. Os autores destacam a importância dos estudos sobre vulnerabilidade, seja na tradição dos estudos ambientais, seja nos estudos sobre saúde ou nos estudos sobre pobreza, para a compreensão e o enfrentamento de questões relacionadas à segurança humana e a sustentabilidade.

O termo vulnerabilidade, muitas vezes, se confunde "com definições anteriores de risco, e da mesma forma, parece ter problema na conexão entre o nível teórico e o operacional" (ROTAVA, 2014, p.32). Esse problema de relação teórico-operacional é também apontado por Berrouet, Machado e Villegas-Palacio (2018), que sinalizam ainda que, diante das degradações e perdas dos serviços ecossistêmicos, uma crescente atenção tem sido dada à análise da vulnerabilidade dos sistemas socioecológicos.

Em se tratando especificamente da vulnerabilidade hídrica, Perveen e James (2011, p.321) conceituam o termo como a "vulnerabilidade de uma região resultante da disponibilidade limitada de recursos hídricos e uso intensivo da água”. Nesse aspecto, Plummer, De Loë e Armitage (2012) realizaram uma síntese das ferramentas de avaliação de vulnerabilidade hídrica existentes, de maneira a facilitar o uso e a adaptação de tais ferramentas a contextos particulares. Os autores identificaram 710 indicadores de $\mathrm{VH}$, categorizados em 22 subdimensões e cinco dimensões (recursos hídricos; outros ambientes físicos; economia; instituições e social).

Da pesquisa dos autores acima citados, a GIRH surge chamando a atenção para uma gama diversificada de valores associados à água e a discussão ilustra até que ponto as ferramentas de avaliação da VH analisadas refletem uma abordagem holística. Esta discussão é também contemplada por Sullivan (2011), que realizou a identificação do estado atual da vulnerabilidade hídrica em escala municipal na África do Sul, e por Hurd et al., (1999), que apresentaram um conjunto de indicadores de vulnerabilidade regional dos usos da água em bacias hidrográficas nos Estados Unidos.

Ainda no país norte-americano, observa-se um crescente interesse pelos estudos de VH a partir da década de 90. Gleick (1990, apud HURD et al., 1999, p.1400) examinou "cinco medidas de vulnerabilidade às mudanças climáticas e as aplicou a cada uma das 18 principais regiões de recursos hídricos dos EUA". No México, Mendoza, Villanueva e Adem (1997) desenvolveram uma avaliação similar em regiões hidrológicas do país. No Brasil, também no início da década de 90, o IPEA adotou cinco estimadores da vulnerabilidade dos sistemas hídricos regionais, realizando ainda análises de vulnerabilidade às secas em cenários de mudanças climáticas (IPEA, 1995).

Essas análises foram desenvolvidas por meio de indicadores compostos de vulnerabilidade no contexto dos recursos hídricos, ferramentas que têm se mostrado úteis para medir o desempenho e a saúde das bacias hidrográficas e sistemas de água. Nessa perspectiva, Gleick $(2015$, p. 2) afirma que "qualquer esforço para avaliar as 
vulnerabilidades dos recursos hídricos será limitado pela escolha de indicadores, pela qualidade e disponibilidade dos dados e por detalhes específicos da região". O autor reforça, desta maneira, a importância em "desenvolver definições, ferramentas e métricas claras que ofereçam maneiras consistentes de categorizar vulnerabilidades relacionadas à água, a fim de identificar pontos de acesso ou riscos regionais e as melhores estratégias para reduzi-los" (GLEICK, 2015, p.2).

Chamando a atenção novamente para o Brasil, salienta-se a relevância de avaliações de $\mathrm{VH}$ especificamente desenhadas para as realidades locais, principalmente de regiões críticas. Apesar de o país apresentar bons índices quando comparado no cenário mundial (Figura 1), as atuais conjunturas reafirmam disparidades históricas em termos da segurança hídrica dos sistemas brasileiros. No ano de 2015, 9\% das cidades brasileiras necessitavam de novas fontes hídricas, e aproximadamente $50 \%$ apresentavam vulnerabilidades associadas à produção de água (ANA, 2019b).

Figura 1: Estresse hídrico no Brasil e no mundo.

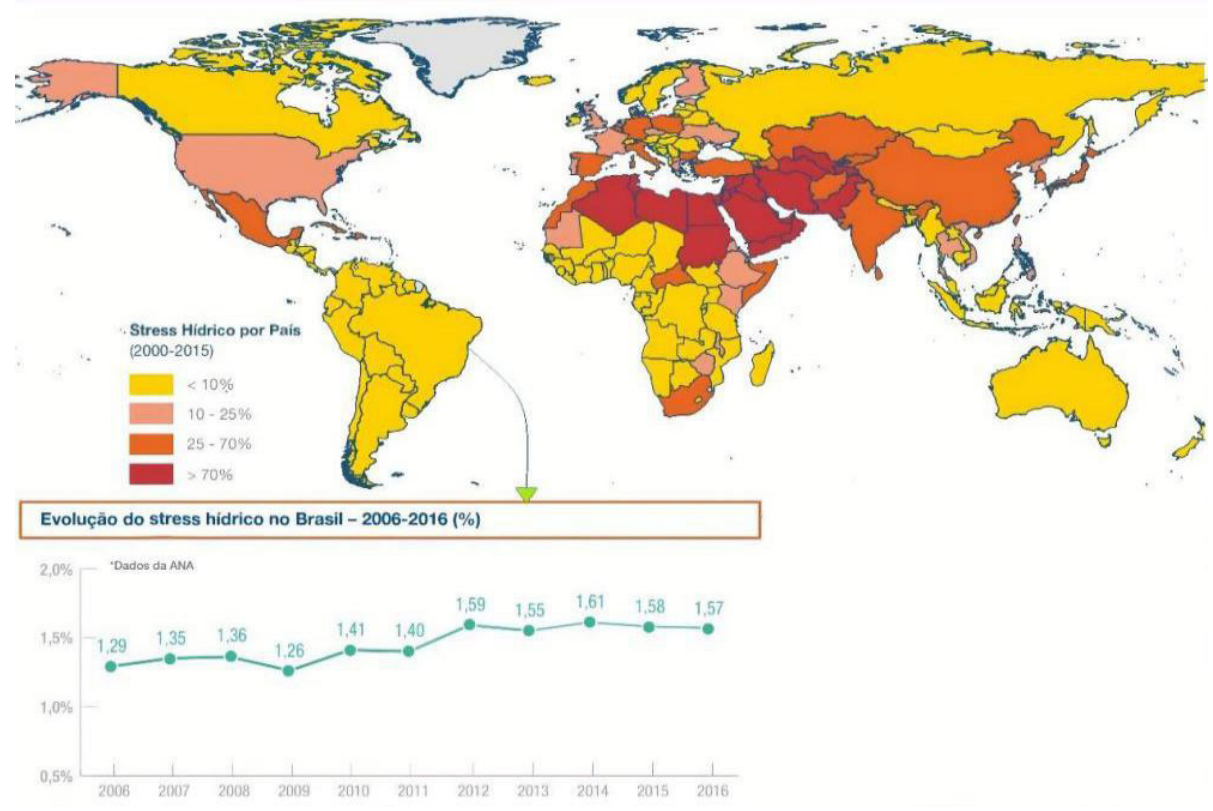

Fonte: ANA (2019b).

\subsection{Mapa conceitual e linha do tempo}

A Figura 2 mostra a estrutura teórica desenvolvida para dar suporte ao Mapa Conceitual relativo à $\mathrm{SH}$ e à $\mathrm{VH}$ na perspectiva da sustentabilidade global. 
Figura 2: Base teórica do mapa conceitual: $\mathrm{SH}$ e VH na perspectiva da sustentabilidade global.

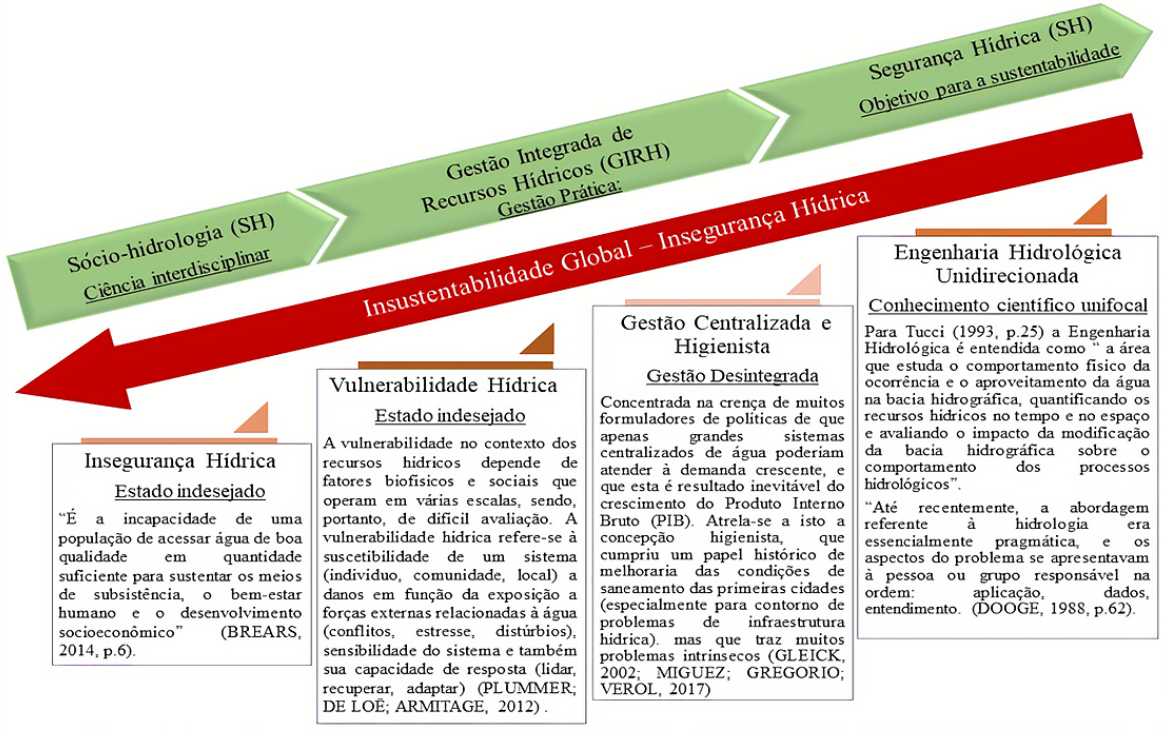

Fonte: Elaboração dos autores.

Baseando-se no conteúdo da Figura 2, destaca-se que o conceito de desenvolvimento sustentável surgiu na década de 1970, chamando atenção para o gerenciamento de recursos naturais do mundo de modo a manter as condições de vida na Terra. No contexto dos recursos hídricos, Marques, Da Cruz e Pires (2015) apontam que os usos desse conceito nos serviços urbanos de água têm aumentado, principalmente aqueles inspirados nas perspectivas do tripé da sustentabilidade, ou Triple Bottom Line (TBL). Conforme Liner e Demonsabert (2011, p.335),

com o tripé da sustentabilidade, os esforços integrados de planejamento de recursos hídricos devem analisar alternativas para abordar as metas potencialmente conflitantes de economia (financeira), ambiental (saúde humana e ecológica) e questões sociais (benefício à humanidade).

Com relação ao cenário atual de sustentabilidade global, abordando especificamente a Agenda 2030 e seus ODS, nota-se, a partir do ODS-6 e de suas metas relacionadas, que a Gestão Integrada de Recursos Hídricos está inserida neste cenário. Segundo Nshimbi (2019), os princípios da Gestão Integrada de Recursos Hídricos visam aumentar a segurança da água por meio da implementação coordenada da gestão. Nesse sentido, estudos recentes têm usado ferramentas e métodos diversos para investigar os impactos da implementação da GIRH para melhorar os benefícios 
econômicos e ambientais em diferentes bacias hidrográficas (AL-JAWAD et al., 2019). Tundisi (2008) reforça a inovação de sistemas de gestão diante do posicionamento central da água, considerando o panorama da sustentabilidade e das mudanças globais, conforme a figura 3 abaixo:

Figura 3: Inovação de Sistemas de Gestão dos recursos hídricos considerando a posição central da água em relação a processos.

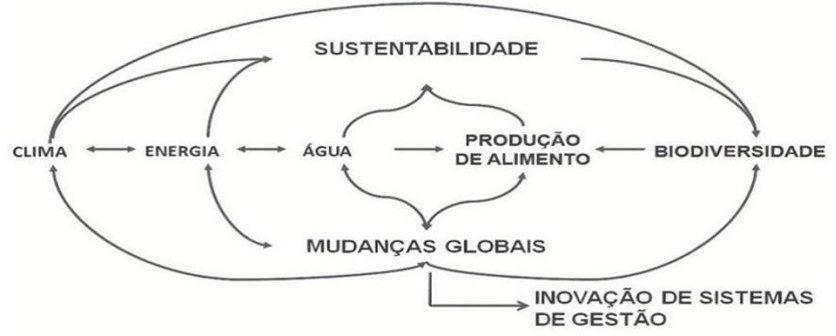

Fonte: Tundisi (2008).

MATRIZ DE COMPONENTES E INTERAÇÕES

Faz-se importante ainda para esta discussão enfatizar as diferenças entre a GIRH e o conceito de Sócio-hidrologia. Massuel (2018, p. 2510) afirma que "a Sóciohidrologia avançou no campo da hidrologia considerando os seres humanos e suas atividades como parte do ciclo da água, e não como fatores externos”. Essa ciência interdisciplinar tem um grande papel no gerenciamento sustentável da água, pois tem o estudo da coevolução dos seres humanos e da água na paisagem como sua essência. Portanto, a prática da GIRH é fundamentalmente apoiada pela ciência da Sóciohidrologia (SIVAPALAN et al., 2014).

$\mathrm{Na}$ mesma dimensão complexa, a segurança hídrica é conceituada por Srinivasan, Konar e Sivapalan (2017, p.12) como um

problema multifacetado, indo além do mero equilíbrio de oferta e demanda. As primeiras tentativas de quantificar a segurança dos recursos hídricos basearam-se em abordagens estáticas pautadas em índices que falharam em reconhecer que a ação humana é intrínseca ao ciclo da água.

Ainda no tocante à Figura 3, Plummer, De Loë e Armitage (2012) afirmam que as avaliações de vulnerabilidade hídrica precisam ser holísticas, de maneira a serem, assim, úteis para os atores e formuladores de políticas de água. Essa afirmação corrobora o disposto na literatura a qual sugere que as práticas de gestão centralizada de cunho fundamentalmente higienista contribuem para que os sistemas estejam mais suscetíveis a danos em função da exposição a forças externas relacionadas à água. As concepções higienistas de gerenciamento hídrico trazem problemas intrínsecos: à 
medida que o foco recai nas consequências da urbanização, inicia-se uma espiral de demandas na qual são aceitos volumes de escoamento cada vez maiores (MIGUEZ; GREGORIO; VEROL, 2017).

Essa disposição administrativa de caráter centralizador teve como alicerce predominante um conhecimento científico unifocal essencialmente pragmático, que, opondo-se às premissas da Sócio-hidrologia, por exemplo, negligenciava a interdisciplinaridade característica da ciência hidrológica (TUCCI, 1993). Salienta-se aqui o disposto por Dooge (1988, p. 64), de que

o desenvolvimento dos recursos hídricos não é simplesmente a aplicação do conhecimento hidrológico a um problema de projeto na engenharia hidráulica. A alocação eficiente e racional de recursos do desenvolvimento da água deve se preocupar também com recursos financeiros e humanos.

No caso brasileiro, os caminhos em destino à insegurança hídrica vinham sendo fortalecidos pelas práticas de gestão centralizadora, nas quais os governos estaduais e federal definiam suas políticas sem que houvesse a participação da sociedade civil, dos usuários de água e dos governos locais (ABERS; JORGE, 2005). Esses tipos de práticas tendem a ser desatentas aos aspectos de justiça social, fator que favorece a propensão para conjunturas de estresse hídrico (WADE, 2018). "O estresse hídrico é o resultado da escassez de água e pode-se manifestar como insegurança na água potável, acesso deficiente, saúde precária, conflito sobre os recursos hídricos, falha na colheita, insegurança alimentar e / ou insegurança energética" (WATERAID, 2012, p.9).

De acordo com Campos e Fracalanza (2010), a descentralização do sistema brasileiro de gestão hídrica seguiu a Constituição Federal de 1988 (BRASIL, 1988) e foi inspirada nos aspectos do modelo francês de gestão participativa, considerando a criação de agências e comitês de bacia. Em termos de políticas públicas para alcance da SH, Passador et al., (2007) configuram uma linha do tempo com três períodos, a saber (Figura 4):

Figura 4: Linha do tempo de Políticas Públicas brasileiras para garantia da SH.

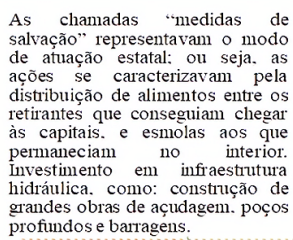

Implementação de programas diversos, tais como: PROTERRA (1971). PROVALE (1972). POLONORDESTE (1974), PROJETO SERTANEJO (1976) e PROHIDRO (1979). Destaca-se que no ano de 1974 foi criada a Companhia de Desenvolvimento dos Vales do São Francisco do Parnaiba do Sâ Francisco e do sucedendo a SUVAIE e tormandose uma empresa pública.

\section{(a)}

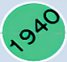

Fonte: Os autores a partir de Passador et al., (2007). 
Considerando-se o exposto, a Figura 5 sintetiza as discussões apresentadas neste artigo e apresenta o Mapa Conceitual para elucidar as abordagens de $\mathrm{SH}$ e de VH na perspectiva da sustentabilidade global.

Figura 5: Mapa conceitual da SH e da VH na perspectiva da sustentabilidade global.

Diferentes focos na definição e no estudo da segurança hídrica:

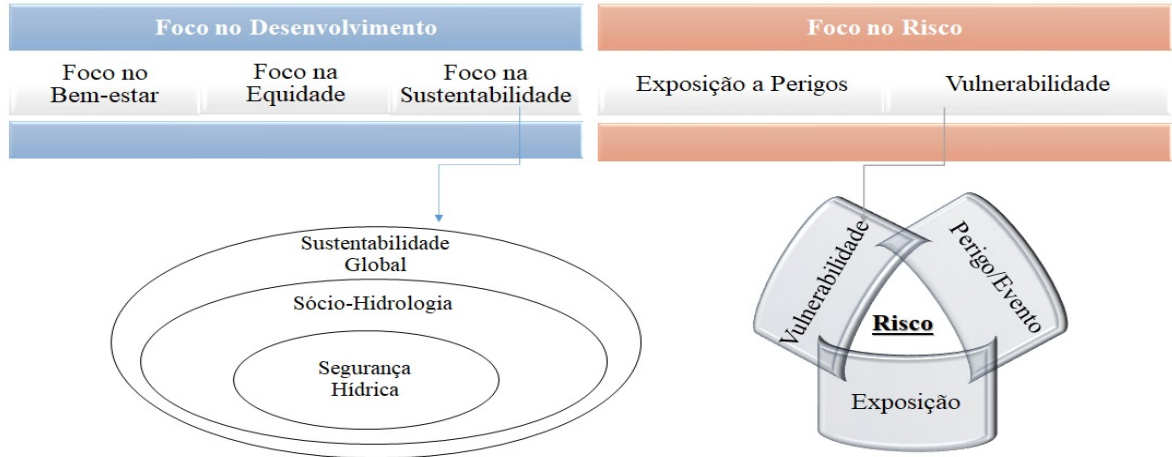

Fonte: Elaboração dos autores, a partir de Hoekstra, Buurman e van Ginkel (2018), Tundisi (2008) e Garrick e Hall (2014).

Segundo Hoekstra, Buurman e Van Ginkel (2018), existem focos distintos na definição e no estudo da segurança hídrica: (i) focos no desenvolvimento e (ii) focos no risco. O (i) foco na sustentabilidade foi o utilizado nesta pesquisa. Nesse sentido, baseando-se em Lew et al., (2016), este estudo concorda que um entendimento prático da perspectiva da sustentabilidade é em termos de seus objetivos principais, que visam, entre outros, a proteção e a manutenção (segurança) dos recursos naturais (e.g. água), enfrentando e mitigando mudanças indesejáveis.

No que se refere aos focos no risco (ii), a abordagem analisada foi a da $v u l$ nerabilidade. De acordo com Garrick e Hall (2014), o risco não é diretamente observável, devendo, portanto, ser avaliado em função de suas componentes perigo, exposição e vulnerabilidade, às quais se conceituam da seguinte forma (GARRICK; HALL, 2014, p.619):

- Perigo - Fenômenos com potencial de causar danos ou prejuízos: secas, inundações, abastecimento/saneamento inadequado, qualidade prejudicial da água;

- Exposição - Pessoas, meios de subsistência, infraestrutura e ativos socioeconômicos que podem sofrer danos causados por eventos perigosos; e

- Vulnerabilidade - Propensão a sofrer danos. 
Conforme Varis, Keskinen e Kummu (2017), quando se trata de segurança hídrica, o tema de vulnerabilidade deve ser considerado, pois pode fornecer enquadramentos e elementos úteis para aprimorar os vínculos entre $\mathrm{SH}$ e desenvolvimento sustentável. Nesse sentido, nota-se a relevância dos conceitos de $\mathrm{SH}$ e VH para o progresso da prática da GIRH em muitos países. Nota-se ainda que o novo paradigma da gestão de recursos hídricos pressupõe que a chamada estrutura de governança, ou institucional, seja analisada como reflexo da cultura, do regime político e organizacional de um país (OECD, 2015).

\section{Considerações finais}

Diante do exposto, observa-se que as evoluções conceituais no campo dos recursos hídricos passaram a contemplar abordagens interdisciplinares com análises socioambientais que, por muito tempo, foram negligenciadas. Especialmente após a eclosão do modelo de GIRH, faz-se cada vez mais necessário compreender as novas concepções no contexto da sustentabilidade e das mudanças globais. Isso se torna relevante não apenas por fornecer as bases para análises mais precisas, mas para que os conceitos possam ser operacionalizados e introduzidos nos projetos de sistemas hídricos e nos processos de governança da água em geral.

O presente artigo apresentou as evoluções conceituais relativas à segurança e à vulnerabilidade hídrica na perspectiva da sustentabilidade, bem como identifica marcos político-institucionais do estado de Pernambuco que guardam relação com o conceito de segurança hídrica. A partir do Mapa Conceitual desenvolvido, conclui-se que há múltiplos focos no conceito e no estudo da $\mathrm{SH}$, sendo aqui analisados os focos na Sustentabilidade e na Vulnerabilidade.

Perante o atual cenário de intensas mudanças globais, a busca pela aplicabilidade prática das premissas do desenvolvimento sustentável se faz cada vez mais primordial e, no contexto da governança hídrica, a natureza inter-relacionada prevista pela Gestão Integrada se mostra coerente com as aspirações em torno da segurança dos recursos hídricos. Dessa forma, nota-se a relevância das discussões expostas neste trabalho para trazer reflexões e orientações sobre questões a serem melhoradas na implementação da GIRH. 


\section{Referências}

ABERS, R.; JORGE, K. D. Descentralização da gestão da água: por que os comitês de bacia estão sendo criados? Ambiente e Sociedade, Campinas, v. 8, n. 2, p. 99-124, 2005. DOI: 10.1590/S1414-753X2005000200006.

ADGER, W.N. Vulnerability. Global Environmental Change, v. 16, n. 3, p. 268-281, 2006. DOI: 10.1016/j.gloenvcha.2006.02.006.

AL-JAWAD, J. Y.; ALSAFFAR, H. M.; BERTRAM, D.; KALIN, R. M. A comprehensive optimum integrated water resources management approach for multidisciplinary water resources management problems. Journal of Environmental Management, v. 239, n. 1, p. 211-224, 2019. DOI: 10.1016/j.jenvman.2019.03.045.

ALMEIDA, L. Q. de. Riscos ambientais e vulnerabilidades nas cidades brasileiras: conceitos, metodologias e aplicações. São Paulo: Cultura Acadêmica, 2012, 215p.

ANA - AGÊNCIA NACIONAL DE ÁGUAS (Brasil). Plano Nacional de Segurança Hídrica (PNSH). Brasília, 2019a. 112 p. ISBN: 978-85-8210-059-2.

ANA - AGÊNCIA NACIONAL DE ÁGUAS (Brasil). ODS 6 no Brasil: visão da ANA sobre os indicadores. Brasília, 2019b. 94 p. il. ISBN:978-85-8210-058-5.

BEEK, E. van; ARRIENS, W. L. Water Security: Putting the Concept into Practice. TEC Background Papers, n. 20. Global Water Partnership: Stockholm, Sweden, p. 1-55, 2014.

BERROUET, L.M.; MACHADO, J.; VILLEGAS-PALACIO, C. Vulnerability of socio-ecological systems: A conceptual Framework. Ecological Indicators, v. 84, p. 632 647, 2018. DOI: 10.1016/j.ecolind.2017.07.051.

BOGARDI, J.J.; DUDGEON, D.; LAWFORD, R.; FLINKERBUSCH, E.; MEYN, A.; PAHL-WOSTL, C.; VÖRÖSMARTY, C. Water security for a planet under pressure: interconnected challenges of a changing world call for sustainable solutions. Current Opinion in Environmental Sustainability, v. 4, n. 1, p. 35-43, 2012. DOI: 10.1016/j. cosust.2011.12.002.

BRASIL. Constituição da República Federativa do Brasil, de 5 de outubro de 1988.

BRASIL. Poder Executivo. Lei no 9.433/1997. Instituiu a Política Nacional de Recursos Hídricos. Diário Oficial da República Federativa do Brasil, Brasília, DF, 09 jan. 1997.

BRASIL. Senado Federal. Projeto de lei do senado no 65, de 2017. Altera a Lei $n^{\circ} 9.433$, de 8 de janeiro de 1997, para incluir a segurança hídrica no âmbito da Política Nacional de Recursos Hídricos. Diário Oficial da República Federativa do Brasil: Brasília, 2017.

BOLSON, S.H.; HAONAT, A.I. A governança da água, a vulnerabilidade hídrica e os impactos das mudanças climáticas no Brasil. Veredas do Direito, Belo Horizonte, v. 13, n. 25, p. 223-248, 2016. DOI: $10.18623 /$ rvd.v13i25.575. 
CARVALHO, J.R.M.; CURI, W.F. Sistema de indicadores para a gestão de recursos hídricos em municípios: uma abordagem através dos métodos multicritério e multidecisor. Revista Brasileira de Gestão e Desenvolvimento Regional, Taubaté, v. 12, n. 2, p. 374-398, 2016.

CIRILO, J. A.; MONTENEGRO, S. M. G. L.; CAMPOS, J. N. B; The Issue of Water in the Brazilian Semi-Arid Region. In: BICUDO, C. E. M.; TUNDISI, J. G.; SCHEUENSTUHL, M. C. B. (Org.). Waters of Brazil - strategic analysis. Editora Springer, p. 59-72, 2017.

CIRILO, J.A. Políticas públicas de recursos hídricos para o semiárido. Estudos avançados, São Paulo, v. 22, n. 63, p. 61-82, 2008. DOI: 10.1590/S0103-40142008000200005.

COOK, C.; BAKKER, K. Water security: Debating an emerging paradigm. Global Environmental Change, v. 22, n. 1, p. 94-102, 2012. DOI: 10.1016/j.gloenvcha.2011.10.011.

CUTTER, S. L. Vulnerability to environmental hazards. Progress in Human Geography, v.20, n.4, p. 529-9, 1996.

DAMACENA, F.D.L. Fundamentos jurídicos para redução da vulnerabilidade hídrica. $\boldsymbol{R} \boldsymbol{e}$ vista Direito Ambiental e sociedade, Caxias do Sul, v. 5, n. 1, p. 54-79, 2015. Disponível: ttp://www.ucs.br/etc/revistas/index.php/direitoambiental/article/view/3841. Acesso em: 24 fev. 2019.

DOOGE, J. C. I. Hydrology in perspective. Hydrological Sciences Journal, v. 33, n. 1, p. 61-85, 2009 (online). DOI: 10.1080/02626668809491223.

DUTRA, M.T.D. Desenvolvimento de um índice de sustentabilidade hidroambiental em bacia hidrográfica: o caso da bacia do rio Capibaribe, Pernambuco. Tese (Doutorado em Engenharia Civil). Universidade Federal de Pernambuco, 2017. 160 f.

GAIN, A. K.; GIUPPONI, C.; WADA, Y. Measuring global water security towards sustainable development goals. Environmental Research Letters, v. 11, P. 1-14, 2016. DOI: 10.1088/1748-9326/11/12/124015.

GARRICK, D.; HALL, J. W. Water Security and Society: Risks, Metrics, and Pathways. Annual Review of Environment and Resources, v. 39, n. 1, p. 611-639, 2014. DOI: /10.1146/annurev-environ-013012-093817.

GLEICK, P. H. Water and Conflict: Fresh Water Resources and International Security. International Security, v. 18, n. 1, p. 79-112, 1993. DOI:10.2307/2539033.

GLEICK, P. H. Soft water paths. Nature. 418: 373. 2002.

GLEICK, P. H. On methods for assessing water-resource risks and vulnerabilities. Environmental Research Letters, v. 10, p. 24-27, 2015. DOI: 10.1088/17489326/10/11/111003. 
GROSBOIS, D.; PLUMMER, R. Problematizing Water Vulnerability Indices at a Local Level: a Critical Review and Proposed Solution. Water Resources Management, v. 29, p. 5015-5035, 2015. DOI:10.1007/s11269-015-1101-0

GWP. Global Water Partnership. Towards Water Security: A Framework for Action. GWP, 2000.

GWP. Global Water Partnership. Water Security: Putting the Concept into Practice, 2014.

GWP-C. Global Water Partnership Caribbean. Sustainability of Integrated Water Resources Management Initiatives in the Caribbean. Global Water Partnership-Caribbean, 2015.

HOEKSTRA, A. Y.; BUURMAN, J.; VAN GINKEL, K. C. H. Urban water security: A review. Environmental Research Letters, v. 13, n. 5, p. 1-15, 2018. DOI: 10.1088/17489326/aaba52.

HOGAN, D. J.; MARANDOLA JR., E. Para uma conceituação interdisciplinar da vulnerabilidade. In: CUNHA, J. M. P. Novas Metrópoles Paulistas - População, vulnerabilidade e segregação. Ed. 1, Campinas: NEPO/UNICAMP, p. 23-50, 2006.

HURD, B.; LEARY, N.; JONES, R.; SMITH, J. Relative regional vulnerability of water resources to climate change. Journal of the American Water Resources Association, 35, n. 6, p. 1399-1409, 1999. DOI:10.1111/j.1752-1688.1999.tb04224.x

IPEA- INSTITUTO DE PESQUISA ECONÔMICA APLICADA. Projeto Áridas Nordeste: Uma Estratégia de Desenvolvimento Sustentável, Brasília, 1995.

JAMES, L.D.; SHAFIEE-JOOD, M. Interdisciplinary information for achieving water security. Water Security, v. 2, p.19-31, 2017. DOI: 10.1016/j.wasec.2017.10.001

JENSEN, O.; WU, H. Urban water security indicators: Development and pilot. Environmental Science \& Policy, v. 83, p. 33-45, 2018. DOI:10.1016/j.envsci.2018.02.003

KRUEGER, E.; RAO, P. S.C.; BORCHARDT, D. Quantifying urban water supply security under global change. Global Environmental Change, v. 56, p. 66-74, 2019. DOI: 10.1016/j.gloenvcha.2019.03.009.

LEW, A. A.; NG, P. T.; NI, C. (NICKEL); WU, T. (EMILY). Community sustainability and resilience: similarities, differences and indicators. Tourism Geographies, v. 18, n. 1, p. 18-27, 2016. DOI:10.1080/14616688.2015.1122664

LINER, B.; DEMONSABERT, S. Balancing the Triple Bottom Line in Water Supply Planning for Utilities. Journal of Water Resources Planning and Management, v. 137, n. 4, p. 335-342, 2011. DOI:10.1061/(asce)wr.1943-5452.0000128

LOPEZ PORRAS, G.; STRINGER, L. C.; QUINN, C. H. Corruption and conflicts as barriers to adaptive governance: Water governance in dryland systems in the Rio del Carmen watershed. Science of The Total Environment, v. 660, p. 519-530, 2019. DOI:10.1016/j.scitotenv.2019.01.030 
MACHADO, F. H. Proposição de indicadores de segurança hídrica: seleção, validação e aplicação na bacia hidrográfica do rio Jundiaí-Mirim, Jundiaí - SP. Tese (Doutorado em Ciências Ambientais). Universidade de São Paulo, 2018. 255 f.

MAO, F.; CLARK, J.; KARPOUZOGLOU, T.; DEWULF, A.; BUYTAERT, W.; HANNAH, D. HESS Opinions: A Conceptual Framework for Assessing Socio-Hydrological Resilience under Change. Hydrol. Earth Syst. Sci, v. 21, p. 3655-3670, 2017. DOI: 10.5194/hess-21-3655-2017

MARANDOLA JR., E.; D'ANTONA, A. de O. Vulnerabilidade: problematizando e operacionalizando o conceito. In: CARMO, R. do; VALÊNCIO, N. (Orgs.) Segurança humana no contexto dos desastres. São Carlos: RiMa Editora, Capítulo 3, p. 45-61, 2014.

MARENGO, J. A. Vulnerabilidade, impactos e adaptação à mudança do clima no semiárido do Brasil. Parcerias Estratégicas, v. 13, n. 27, p. 149-176, 2008. Disponível em: http://seer.cgee.org.br/index.php/parcerias_estrategicas/article/view/329. Acesso em: 20 ago. 2019.

MARENGO, J.A.; ALVES, L.M.; BESERRA, E.A.; LACERDA, F.F. Variabilidade e Mudanças Climáticas no Semiárido. In: MEDEIROS, S.S., GHEYI, H.R., GALVÃO, C.O., PAZ, V.P. (eds). Recursos Hídricos em regiões áridas e semiáridas. INSA, Campina Grande, p. 383-416, 2011.

MARQUES, R. C.; DA CRUZ, N. F.; PIRES. Measuring the sustainability of urban water services. Environmental Science \& Policy, v. 54, p. 142-151, 2015. DOI:10.1016/j.envsci.2015.07.003

MASSUEL, S., RIAUX, J., MOLLE, F., KUPER, M., OGILVIE, A., COLLARD, A.-L., BARRETEAU, O. Inspiring a Broader Socio-Hydrological Negotiation Approach with Interdisciplinary Field-Based Experience. Water Resources Research, v. 54, n. 4, p. 2510-2522, 2018. DOI:10.1002/2017wr021691

MELO, M.C.; FORMIGA-JOHNSSON, R.M. O conceito emergente de segurança hídrica. Sustentare, v. 1, n. 1, p.72-92, 2017. DOI: http://dx.doi.org/10.5892/st.v1i1.4325.

MENDOZA, V. M.; VILLANUEVA, E. E.; ADEM, J. Vulnerability of basins and watersheds in Mexico to global climate change. Climate Research, v. 9, p. 139-145, 1997. DOI: $10.3354 /$ cr009139.

MIGUEZ, M.G.; DI GREGORIO, L.T.; VERÓL, A.P. Gestão de Riscos e Desastres Hidrológicos. Rio de Janeiro: Elsevier Editora Ltda., v. 1. 368 p., 2017.

NSHIMBI, C.C. SDGs and decentralizing water management for transformation: Normative policy coherence for water security in SADC river basin organizations. Physics and Chemistry of The Earth, Parts A/b/c, v. 111, p.1-12, 2019. Elsevier BV. DOI: 10.1016/j.pce.2019.02.010.

OECD. Water Security for Better Lives. OECD Studies on water. OECD Publishing, 2013. DOI: $10.1787 / 9789264202405$-en 
PASQUAL, J.C.; LARDIZABAL, C.C.; HERRERA, G. Water-Energy-Food Nexus: Comparative Scenarios and Public Policy Perspectives from Some Latin American Countries Regarding Biogas from Agriculture and Livestock. Journal of Agricultural Science and Technology A, v. 5, n. 6, p. 408-427, 2015. DOI: 10.17265/21616256/2015.06.004

PASQUAL, J.C.; BOLLMANN, H.A.; SCOTT, C. Water-Energy-Food Nexus: Background and Perspectives for Brazil and the United States by 2050. Journal of Agricultural Science and Technology B, v. 6, n. 2, p. 108-120, 2016. DOI: 10.17265/2161$6264 / 2016.02 .006$

PASSADOR, C. S.; PASSADOR, J.L.; ARRAES, A.M.D.; ARRAES, H.F.L. Políticas Públicas de Combate à Seca no Brasil e a Utilização das Cisternas nas Condições de Vida de Famílias na Região do Baixo Salitre (Juazeiro - BA): uma Dádiva de Deus? In: XXXI EnANPAD 2007, 2007, Rio de Janeiro. Anais [...] XXXI EnANPAD 2007, 2007. v. 1. p. 1-15.

PERVEEN, S.; JAMES, L. A. Scale invariance of water stress and scarcity indicators: Facilitating cross-scale comparisons of water resources vulnerability. Applied Geography, v. 31, n. 1, p. 321-328, 2011. DOI:10.1016/j.apgeog.2010.07.00

PLUMMER, R.; DE LOË, R.; ARMITAGE, D. A Systematic Review of Water Vulnerability Assessment Tools. Water Resources Management, v. 26, n. 15, p. 4327-4346, 2012. DOI:10.1007/s11269-012-0147-5

RASUL, G.; SHARMA, B. Water, Food, and Energy Nexus in South Asia: Implications for Adaptation to Climate Change. Handbook of Climate Change Adaptation, p.1-18, 2014. DOI: 10.1007/978-3-642-40455-9_81-1

RIBEIRO, N. B.; FORMIGA-JOHNSSON, R. M. Discussões sobre governança da água: tendências e caminhos comuns. Ambiente \& Sociedade, v. 21, 2018. DOI:10.1590/ 1809-4422asoc0125r2vu1811ao

RIBEIRO, C.R.; PIZZO, H.S. Avaliação da sustentabilidade Hídrica de Juiz de Fora MG. Revista de Geografia da UFC, v. 10, n. 21, p. 171-188, 2011.

ROTAVA, J. Índices de resiliência hídrica e de perigo para gestão do risco de inundações urbanas. Dissertação (Mestrado em Hidráulica e Saneamento). Escola Politécnica de São Carlos, Universidade de São Paulo. 2014. 133 f.

SAVENIJE, H. H. G.; VAN DER ZAAG, P. Integrated water resources management: Concepts and issues. Physics and Chemistry of the Earth, Parts A/B/C, v.33, n.5, p. 290-297, 2008. DOI:10.1016/j.pce.2008.02.003

SIVAPALAN, M.; SAVENIJE, H. H. G.; BLÖSCHL, G. Socio-hydrology: A new science of people and water. Hydrological Processes, v. 26, n.8, p.1270-1276, 2012. DOI:10.1002/ hyp. 8426 
SIVAPALAN, M.; KONAR, M.; SRINIVASAN, V.; CHHATRE, A.; WUTICH, A.; SCOTT, C. A.; WESCOAT, J. L.; RODRÍGUEZ-ITURBE, I. Socio-hydrology: Use-inspired water sustainability science for the Anthropocene. Earth's Future, v. 2, n. 4, p. 225-230, 2014. American Geophysical Union (AGU). DOI: 10.1002/2013ef000164.

SRINIVASAN, V.; KONAR, M.; SIVAPALAN, M. A dynamic framework for water security. Water Security, v.1, p. 12-20, 2017. DOI:10.1016/j.wasec.2017.03.001

SULLIVAN, C.A. Quantifying water vulnerability: a multi-dimensional approach. Stochastic Environmental Research and Risk Assessment, v.25, n.4, p. 627-640, 2011. DOI: $10.1007 / \mathrm{s} 00477-010-0426-8$

TUCCI, C. E. M. (Org.). Hidrologia: ciência e aplicação. Porto Alegre: Editora da Universidade, 1993. 943p.

TUCCI, C. E. M. Urbanization and water resources. In: BICUDO, C. E. D. M.; TUNDISI, J. G. e SCHEUENSTUHL, M. C. B. (Ed.). Water of Brazil: strategic analysis. Switzerland: Springer International Publishing, 2017. p. 89-104. ISBN 978-3-319-41372-3.

TUNDISI, J.G. Recursos hídricos no futuro: problemas e soluções. Estudos Avançados, São Paulo, v. 63, n. 22, p.7-16, 2008

UN- UNITED NATIONS. General Assembly Resolution A/RES/70/1. Transforming Our World, the 2030 Agenda for Sustainable Development, 2015.

UN WATER. Water Security \& the Global Water Agenda - A UN-Water Analytical Brief, 2013.

UNESCO. International Hydrological Programme (IHP), Eighth Phase, Water Security: Responses to Local, Regional and Global Challenges. Strategic Plan IHP-VIII (20142021). Paris, 2013.

VAN NOORDWIJK, M.; KIM, Y.-S.; LEIMONA, B.; HAIRIAH, K.; FISHER, L. A. Metrics of water security, adaptive capacity, and agroforestry in Indonesia. Current Opinion in Environmental Sustainability, v. 21, p. 1-8, 2016. DOI:10.1016/j.cosust.2016.10.004

VARIS, O; KESKINEN, M.; KUMMU, M. Four dimensions of water security with a case of the indirect role of water in global food security. Water Security, v.1, p. 36-45, 2017. DOI:10.1016/j.wasec.2017.06.002

VÖRÖSMARTY, C. J.; OSUNA, V. R.; CAK, A. D.; BHADURI, A.; BUNN, S. E.; CORSI, F.; UHLENBROOK, S. Ecosystem-based water security and the sustainable development goals. Ecohydrology \& Hydrobiology, v. 18, n.4, p. 317-333, 2018. DOI:10.1016/j. ecohyd.2018.07.004

WADE, S. Is water security just? Concepts, tools and missing links. Water International, v.43, n.8, p.1026-1039, 2018. DOI:10.1080/02508060.2018.1543750 
WANG, Q.; LI, S.; LI, R. Evaluating water resource sustainability in Beijing, China: Combining PSR model and matter-element extension method. Journal of Cleaner Production, v.16, n.1, p. 171-179, 2018. DOI:10.1016/j.jclepro.2018.09.057

WATERAID. Water security framework. London: WaterAid, 2012.

WWC-WORLD WATER COUNCIL. A Pact for a water security world - 2013- 2015 Strategy. 2012.

YANG, F.; SHAO, D.; XIAO, C.; TAN, X. Assessment of urban water security based on catastrophe theory. Water Sci. Technol, v.66, n.3, p. 487-493, 2012. DOI: 10.2166/ wst.2012.182 
